
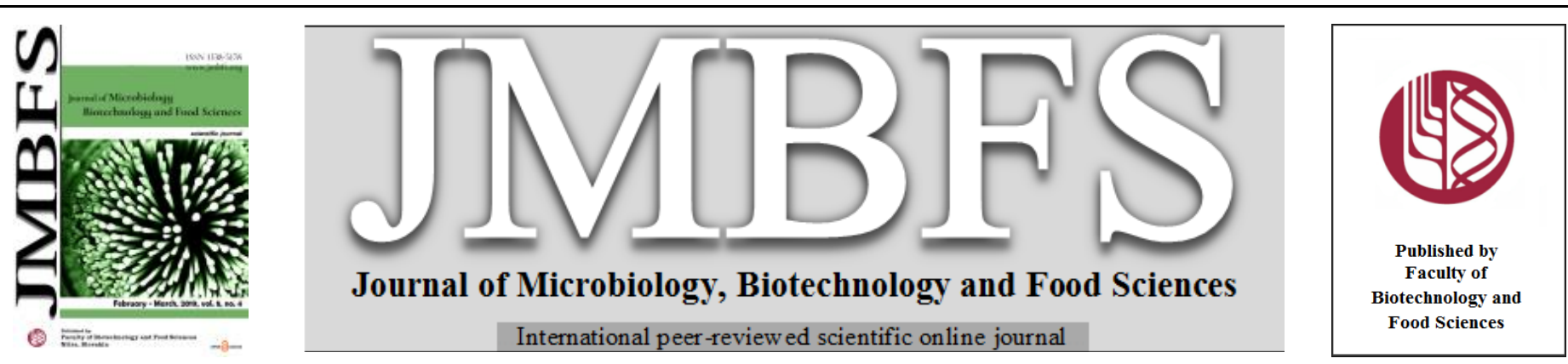

\title{
MOLECULAR MODELING AND IN SILICO CHARACTERIZATION OF NITROGEN METABOLITE REPRESSOR NmrA OF OPPORTUNISTIC HUMAN PATHOGEN Aspergillus fumigatus
}

\author{
Muhammad Tausif Chaudhry*, Raheela Chaudhry \\ Address(es): \\ CMD, National Physical and Standards Laboratory, PCSIR, Islamabad, Pakistan.
}

*Corresponding author: tausif_chaudhry@yahoo.com

doi: 10.15414/jmbfs.2019.8.5.1169-1173

\section{ARTICLE INFO}

Received 15. 11. 2018

Revised 15. 12. 2018

Accepted 29. 12. 2018

Published 1. 4. 2019

Regular article

open $\partial_{\text {ACCESS }}$

\begin{abstract}
The most prevalent airborne human pathogenic fungus Aspergillus fumigatus is ubiquitous in soil and like other ascomycotous fungi it can utilize a variety of nitrogen sources. Nitrogen metabolite repression (NMR) may induce virulence in A. fumigatus as in pathogenic fungi but the mechanism of regulation and its key components are not identified. The present study focuses on the molecular modeling and in silico characterization of the main player of this regulation, the NmrA of A. fumigatus. Physiochemical and structural characterization using various sequence and structure based predictors and quality assessment of the proposed two- and threedimensional models were carried out. The characteristic motifs such as glycine-rich $\mathrm{NAD}(\mathrm{P})$-binding motif $(G \times \mathrm{xx} G \times \mathrm{xx} G)$ and altered active site motif $(H \mathrm{xxxK})$ were located in NmrA along with DNA-binding residues (T11, R39, D40 and A45). The results obtained using bioinformatics tools indicated that the protein was hydrophilic in nature, stable in vitro and had very low disorder probability. Based on the quality score, the proposed secondary and tertiary structures were correct and extremely good to represent NmrA of A. fumigatus. Phylogenetic analysis signified its close relation with NMR regulatory protein of opportunistic human pathogens A. lentulus and A. novofumigatus.
\end{abstract}

Keywords: Aspergillus fumigatus; conserved motif; in silico; nitrogen metabolite repression; NmrA; phylogenetic analysis; tertiary structure

\section{INTRODUCTION}

The opportunistic human pathogen, Aspergillus fumigatus is a ubiquitous saprophytic fungus, which is associated with various diseases of the respiratory tract. Its airborne spores (conidia) are highly dispersible and significantly more hydrophobic than those of other Aspergillus species (Kwon-Chung and Sugui, 2013). Invasive aspergillosis caused by colonization of these conidia in patients with immune defects causes more than 0.4 million deaths each year (Bongomin et al., 2017). Likewise, its colonization in respiratory airways develops allergic bronchopulmonary aspergillosis (ABPA) with serious consequences in about 5 million asthmatic people worldwide (Denning et al., 2014).

Like other ascomycotous fungi, A. fumigatus grows in soil recycling nitrogen and carbon by degrading organic debris rich in polysaccharides and proteinacious substrates (Latge 1999; Abad et al., 2010). Nitrogen limitation is reported to induce the expression of virulence genes in various pathogenic fungi (LopezBerges, 2010). In filamentous fungi, nitrogen metabolite repression (NMR) occurs when less-preferred nitrogen sources (such as nitrate) are simultaneously available in the medium with energetically favored compounds (ammonium or glutamine) but the pathway for non-preferred nitrogen sources remains inactive (Tudzynski, 2014). NMR is a global regulatory switch which controls activation of enzyme and permease coding genes for degradation of non-preferred nitrogen sources (Magasanik and Kaiser, 2002). As reported, the major GATA transcription factor AreA and its co-repressor NmrA play a vital role in nitrogen regulatory network (Tudzynski, 2014). Inactivation of these regulatory proteins results in inability of fungal growth on nitrogen sources except for ammonium and glutamine (Wilson and Arst, 1998).

NmrA is among the highly conserved regulatory proteins among Aspergillus species (Lamb et al., 2003). The regulator gene $n m r A$ has been characterized in two Aspergillus species; A. nidulans (Andrianopoulos et al., 1998) and A. flavus (Han et al., 2016). NmrA of A. nidulans was monomeric in solution when overexpressed in E. coli and the protein was crystallized into trigonal, monoclinic and hexagonal forms (Nichols et al., 2001). Interestingly, instead of carbon catabolite repressor CreA, NmrA controls the ability of both GATA transcription factors AreA and AreB to sense the status of carbon metabolism (Macios et al., 2012) $\mathrm{NmrA}$ is a member of the short-chain dehydrogenase reductase (SDR) superfamily. Like other SDR family proteins, it binds to dinucleotides $\mathrm{NAD}^{+}$and $\mathrm{NADP}^{+}$. However, it has a decreased affinity for the reduced forms NADH and NADPH that indicates its possible role in redox signaling, and no affinity for nitrogen metabolite signal molecules ammonium and glutamine (Lamb et al., 2003). In addition to preventing activation of AreA, $n m r A$ in A. flavus appears to be involved in aflatoxin biosynthesis, conidiation, sclerotia formation, invasive virulence and susceptibility to rapamycin and methanesulfonate.

As evident from the comparative genomic analysis of sequenced genera, $A$ fumigatus genes are different than those commonly found in other pathogenic fungi i.e. Aspergillus, Candida and Cryptococcus (Nierman et al., 2005). Nitrogen limitation may induce expression of virulence genes in A. fumigatus like other pathogenic fungi. However, nitrogen metabolite repression in A. fumigatus has not yet characterized. Moreover, the components of this regulation and their structures are not identified. The present study is designed to propose the twoand three-dimensional structures of NmrA of A. fumigatus using homology modeling and its in silico characterization using various bioinformatics tools. The structural validation and quality of the proposed model was assessed by Ramachandran plot and some neural network based predictors. Conservations and characteristic motifs were also located in NmrA. Important physiochemical parameters were computed using protein sequence and structural features like disorder, foldability and bonds of the proposed structures were predicted. In addition, the evolutionary distances were computed and phylogenetic analysis was conducted for nineteen related Aspergillus species.

\section{MATERIAL AND METHODS}

Sequence analysis and alignments

Protein sequence of NmrA (Accession No. KEY 83585) was retrieved from complete genome sequence of A. fumigatus var. RP-2014 available at GenBank (http://www.ncbi.nlm.nih.gov/genbank) with accession No. GCA 000731615 This sequence was compared with a library of database of sequences using the most widely used bioinformatics program for protein sequence searches, basic local alignment tool for protein, BLASTP (Altschul et al., 1990), at NCBI server to get most similar protein sequences. Similarly, protein sequences of NmrA 
from eighteen other Aspergillus species were retrieved. NCBI's conserved domain database (CDD; Marchler-Bauer et al., 2017) was used to annotate functional units and conservations in NmrA. Pair-wise and multiple sequence alignments were made with CLUSTAL X 2.1 (Larkin et al., 2007). Sequence identity was determined using BioEdit 7.2.5 (Hall, 1999).

Secondary and tertiary structure modeling

A specific distribution of $\alpha$-helices, $\beta$-sheets and $3_{10}$ helices in the secondary structure of NmrA was predicted by SSPro8 at SCRATCH protein predicto server (Cheng et al., 2005). Similarly, tertiary structure was predicted by 3DPro at SCRATCH server. The final three-dimensional structures of NmrA were produced using the program VMD 1.9.3 (Humphrey et al., 1996).

Physiochemical characterization and structural features

Physiochemical parameters like molecular weight, isoelectric point (pI), mola attenuation coefficient at $280 \mathrm{~nm}$, instability index, aliphatic index and gran average of hydropathicity were computed by ProtParam (Gasteiger et al., 2003) Structure features like disordered residues, disorder probability, number of domains and presence of disulfide bonds were determined by SCRATCH (Cheng et al., 2005). Unfoldability was predicted by FoldIndex (Prilusky et al., 2005) Mean hydrogen bond energy and percentage of packing defects were determined by VADAR 1.8 (Willard $\boldsymbol{e}$ t al., 2003). A structural based neural network predictor DISPLAR (Tjong and Zhou, 2007) was used to predict NmrA residues that contact DNA.

Structure validation

Ramachandran plot was drawn for structure validation, which is considered the most sensitive way to assess the overall quality of protein structure in the absence of experimental data (Hollingsworth and Karplus, 2010). The plot calculations were computed by PROCHECK (Laskowski $\boldsymbol{e t}$ al., 1993) that evaluates stereochemical quality of the predicted structure and emphasizes disallowed regions. Similarly, a neural network based predictor ProQ (Wallner and Elofsson, 2003) was used to predict quality of $\mathrm{NmrA}$ model. Another program QMEAN (Benkert et al., 2009) was used that assigns a quality score which is used to rank the predicted structure.

Identification of NmrA accessory proteins

NmrA protein sequences from nineteen Aspergillus species were retrieved from NCBI protein database (www.ncbi.nlm.nih.gov/protein). To predict proteinprotein functional interactions and to calculate confidence score of all the interactions, STRING 10.5 (Szklarczyk et al., 2017) was used.

Phylogenetic analysis

The evolutionary history was inferred using the neighbor-joining method of Saito and Nei (1987) based on 1000 replications. The evolutionary distances among NmrA from nineteen Aspergillus species including A. fumigatus were computed using the Poisson-correction (PC) method (Zuckerkandl and Pauling, 1965 with the units of the number of amino acid substitutions per site using MEGA7 (Kumar et al., 2016)

\section{RESULTS AND DISCUSSION}

In the most prevalent airborne pathogenic fungus A. fumigatus, NmrA is repressor of the GATA transcriptional factor AreA, which regulates several genes required for utilization of less-preferred nitrogen sources such as nitrate (Tudzynski, 2014). Conversely, the bZIP protein MeaB is an activator of NmrA (Wong et al., 2007). These three regulatory proteins NmrA, AreA and MeaB are highly conserved in filamentous fungi (Wagner et al., 2010). NmrA of $A$. fumigatus has not been characterized on molecular bases and the regulatory components are not identified. The present study elucidates the structural modeling and in silico characterization using bioinformatics tools.

\section{Secondary structure of NmrA}

As predicted by SSPro8 (Cheng et al., 2005), NmrA of A. fumigatus consisted of $11 \alpha$-helices, $11 \beta$-sheets, and two $3_{10}$ helices (Figure 1). Conserved amino acid residues in the selected nineteen Aspergillus species are highlighted in black. Moreover, dinucleotide $\mathrm{NADP}^{+}$binding site, active site and regulatory sites are marked in the protein sequence. As reported, the characteristic nucleotidebinding motif in bi-domain SDRs is $G \times x G \times x G$ (Mulichak et al., 1999). This glycine-rich motif was located in NmrA (amino acid residues 9-15). Similarly, the SDR characteristic motif $H \mathrm{xxx} K$ (residues 133-137) was present instead of $Y \mathrm{xxx} K$, which is associated with enzyme active site (Stammers $\boldsymbol{e t}$ al., 2001). NmrA binding to the regulator AreA blocks it from binding the DNA.

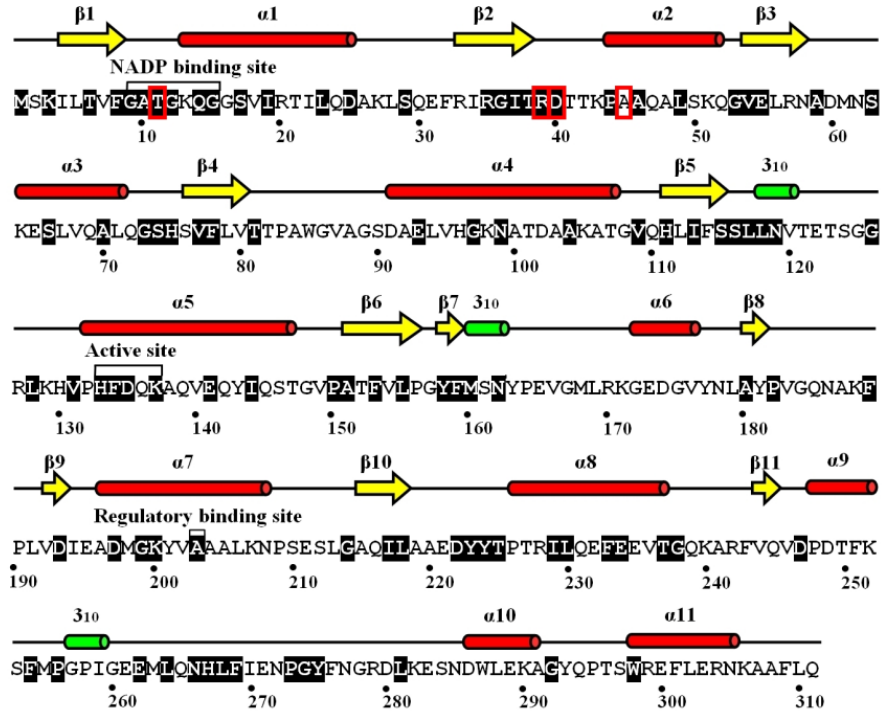

Figure $1 \mathrm{~A}$ specific distribution of $\alpha$-helices, $\beta$-sheets and $3_{10}$ helices in the secondary structure of NmrA of A. fumigatus as predicted by SCRATCH. The characteristic nucleotide-binding glycine-rich motif $(G \times x G \times x G)$, characteristic SDR motif associated with active site $(H \mathrm{xxx} K)$ and regulatory binding site are labeled. Four DNA binding residues $(T 11, R 39, D 40$ and $A 45)$ predicted by DISPLAR are shown in red boxes. Residues conserved in selected nineteen Aspergillus species are highlighted in black.

\section{Tertiary structure of NmrA}

A tertiary structure of NmrA of A. fumigatus is shown in Figure 2. The overal structure of NmrA consisted of two domains. The $\mathrm{N}$-terminal domain contains six parallel $\beta$-sheets (strands $\beta-1$ to $\beta-6$ ) interconnected by $\alpha$-helices ( $\alpha-1$ to $\alpha-5$, residues $1-156)$ and a $3_{10}$ helix. The $\mathrm{C}$-terminal domain contains five $\beta$-sheets (strands $\beta-7$ to $\beta-11)$, six $\alpha$-helices $(\alpha-6$ to $\alpha-11)$ and two $3_{10}$ helices. A structurally diverse loop region of 32 amino acid residues between $\alpha-9$ and $\alpha-10$ (residues 253-285) was also located. This region was found to be disordered to varying degrees in NmrA of A. nidulans and C-terminal domain was required for full biological activity (Stammers et al., 2001).
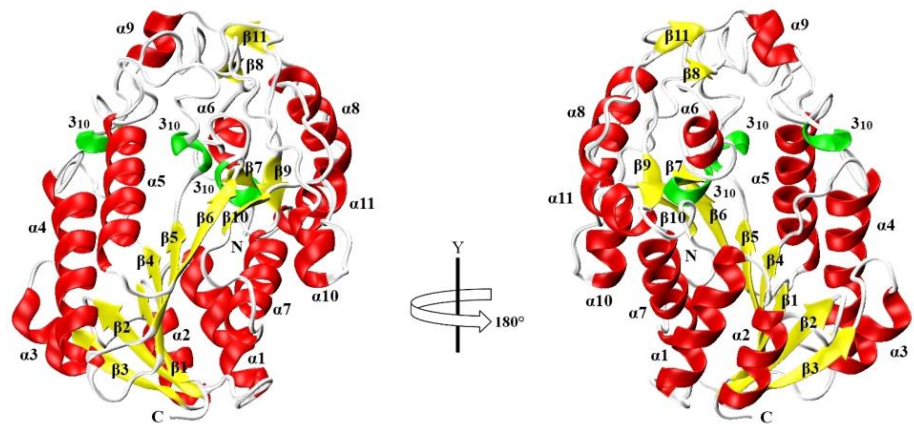

Figure 2 Tertiary structure of NmrA of A. fumigatus before and after $180^{\circ}$ rotation along y-axis. The $\alpha$-helices are shown in red color while $\beta$-sheets and $3_{10}$ helices as yellow arrows and small green helices, respectively. The C- and $\mathrm{N}$ terminal are indicated by $\mathrm{C}$ and $\mathrm{N}$, respectively in the structure drawn by VMD

\section{Physiochemical characterization}

The physiochemical parameters of NmrA of A. fumigatus as determined by ProtParam are shown in Table 1. At pH 5.92 i.e. pI of the protein, net charge on NmrA is zero. Instability index is used to determine whether the proteins will be stable in test tube. Values $<40$ indicated that protein was fairly stable (Guruprasad et al., 1990). The instability index 37.28 indicated that mrA was stable in vitro. As defined, aliphatic index (AI) of a protein is the relative volume occupied by aliphatic side chains of non polar and hydrophobic alanine, isoleucine, leucine proline and valine (Ikai, 1980). 106 of total 311 amino acid residues $(\sim 34 \%)$ were aliphatic in NmrA and an index of 37.28 was fairy expected for this protein of mesophilic origin. Grand average of hydropathy (GRAVY) values are calculated by dividing sum of hydropathy values of all the amino acid residues by total number of residues (Gasteiger et al., 2003). As poteins with GRAVY values $\leq 0$ are hydrophilic, a value of -0.338 indicated the hydrophilic nature of NmrA. 
Table 1 Global sequence features of NmrA of A. fumigatus computed by ProtParam.

\begin{tabular}{lc}
\hline Parameter & Calculated Value \\
\hline Amino acid residues & 311 \\
Molecular weight & $34210.578 \mathrm{kDa}$ \\
Isoelectric point $(\mathrm{pI})$ & 5.92 \\
Molar attenuation coefficient $(\varepsilon)$ at 280 & $31400 \mathrm{M}^{-1} \mathrm{~cm}^{-1}$ \\
nm & 37.28 \\
Instability index & 78.75 \\
Aliphatic index (AI) & -0.338 \\
Grand average of hydropathy index & \\
(GRAVY) & \\
\hline
\end{tabular}

\section{Structural features}

Structural features of NmrA are shown in Table 2. A wide range of biological processes such as gene regulation, DNA replication and repair are controlled by protein-DNA interactions. Four amino acid residues in NmrA sequence were predicted by DISPLAR (Tjong and Zhou, 2007) to bind DNA. These were $T 11$, $R 39, D 40$ and A45. These residues are also highlighted in Figure 1. Other 196 residues did not participate in DNA binding and 111 residues were buried inside protein molecule. As NmrA of A. fumigatus does not contain any cysteine residues, there were no disulfide bonds. Due to the presence of only one disordered residue, the disorder probability ws very low $(0.32 \%)$. As determined by VADAR (Willard et al., 2003), $z$ score values for mean hydrogen bond energy, percentage of packing defects and percentage of bad bond length were $0.98,1.93$ and 0.49 , respectively.

Table 2 Structural features of NmrA of A. fumigatus determined by SCRATCH, FoldIndex and VADAR.

\begin{tabular}{lc}
\hline Parameter & Calculated Value \\
\hline Number of domains & 1 \\
Disordered residues & 1 \\
Disorder probability & $0.32 \%$ \\
Disufide bonds & 0 \\
Packing defects & 88 \\
Unfoladability & 0.126 \\
Free energy of folding & $-288.51 \mathrm{~kJ} \mathrm{~mol}^{-1}$ \\
\hline
\end{tabular}

\section{Structure validation}

The overall quality of NmrA structure was assessed by the distribution of two torsion angles $\varphi$ and $\psi$, usually called the Ramachandran plot (Hollingsworth and Karplus, 2010) and the plot calculations were computed by PROCHECK As shown in Figure 3A, about $83 \%$ of amino acid residues (221 of 311) lie in the most favored or core regions (shown in red) that confirmed good quality of the proposed structure. Also, $12 \%$ and $4.5 \%$ residues were in additional allowed regions (shown in yellow) and generously allowed regions (shown in light yellow), respectively. Only two residues, $L 27$ and $A 308$ lie in disallowed region as highlighted in plot. As predicted by the neural network based predictor ProQ, the Levitt-Gerstein (LG) score (Levitt and Gerstein, 1998) and MaxSub score (Siew et al., 2000) were 4.053 and 0.352 , respectively. LG score $>4$ indicated extremely good model and MaxSub score $>0.1$ shows correct model (Cristobal et al., 2001). Thus, the proposed structure was correct and extremely good to represent NmrA of A. fumigatus. Similarly, score computed by QMEAN was 0.703 with $z$ score value -1.08 as shown in comparison with non-redundent set of protein data bank (PDB) structures (Figure 3B), which indicates good quality of the protein structure.

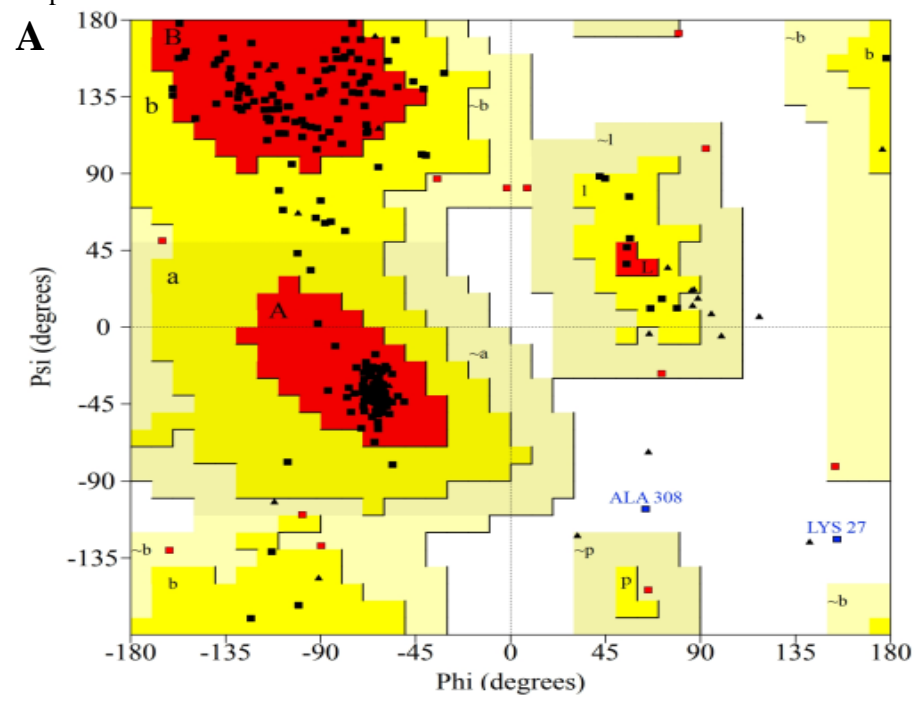

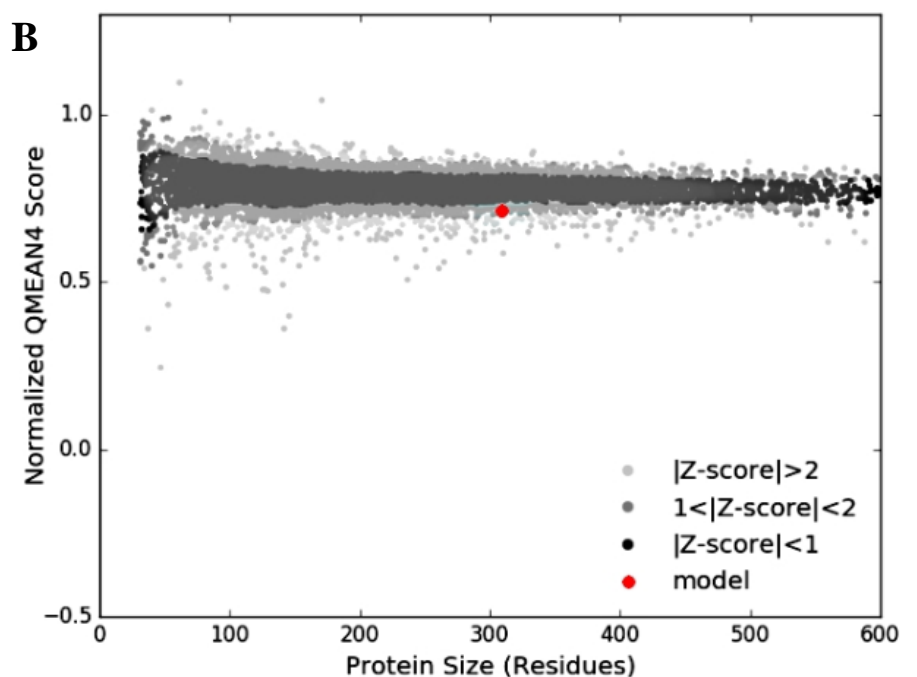

Figure $3 \mathrm{NmrA}$ structure validation. (A) Ramachamdaran plot produced by PROCHECK. (B) Quality score of NmrA computed by QMEAN. The plot shows comparison with non-redundant set of PDB structures.

\section{Identification of NmrA accessory proteins}

The accessory interacting proteins accompany and assist the primary protein for its function or help it to fold, stabilize and locate in the right place. Eleven accessory proteins of NmrA (KEY83585) of A. fumigatus were identified by STRING database. The confidence interval map of these proteins is shown in Figure 4. As indicted by Table 3 these proteins were involved in biosynthesis and metabolism of amino acids and secondary metabolites in A. fumigatus. Confidence score of the accessory protein glutamate synthase Glt1 (KEY80835) as identified was 0.424

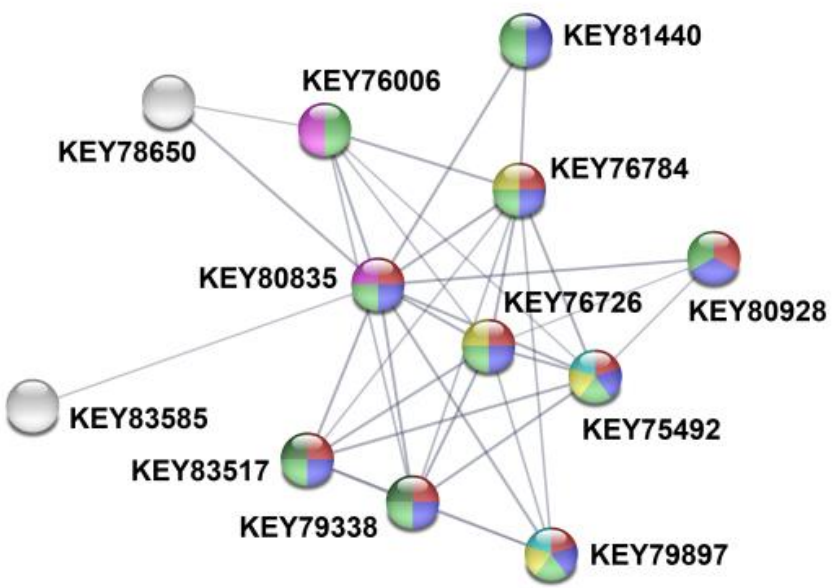

Figure 4 Confidence interval map of the eleven accessory proteins of NmrA that play an important role in nitrogen metabolite repression in A. fumigatus. The interaction network was analyzed by STRING.

Table 3 KEGG pathways of the accessory proteins of NmrA of A. fumigatus identified by STRING. The colors correspond to Figure 4.

\begin{tabular}{lccc}
\hline Pathway ID & Description & $\begin{array}{c}\text { Count } \\
\text { protein set }\end{array}$ & in \\
\hline 01100 & $\begin{array}{c}\text { Metabolic pathways } \\
\text { Biosynthesis of secondary } \\
\text { metabolites }\end{array}$ & 9 & 8 \\
01110 & $\begin{array}{c}\text { Biosynthesis of amino } \\
\text { acids }\end{array}$ & 8 & \\
01230 & $\begin{array}{c}\text { Phenylalanine, Tyrosine } \\
\text { and Tryptophan } \\
\text { biosynthesis } \\
00400\end{array}$ & 4 & \\
00250 & $\begin{array}{c}\text { Alanine, Aspartate and } \\
\text { Glutamine metabolism }\end{array}$ & 2 & \\
00340 & $\begin{array}{c}\text { Histidine metabolism } \\
\text { Glycine, Serine and } \\
\text { Threonine metabolism }\end{array}$ & 2 & \\
00260 & & 2 & \\
\hline
\end{tabular}




\section{Phylogenetic analysis}

Figure 5 represents the phylogenetic relationship of NmrA of A. fumigatus and other nitrogen metabolite regulatory proteins from eighteen Aspergillus species. Phylogenetically close species were also opportunistic human pathogens that cause invasive aspergillosis with high mortality rates i.e. A. lentulus and $A$ novofumigatus (Balajee et al., 2005; Peláez et al., 2013) with 91.3 and $89 \%$ sequence identity, respectively. NmrA of Histoplasma capsulatum, a dimorphic fungus and causative agent of pulmonary histoplasmosis had only $45.5 \%$ sequence identity and was taken as out group. Three other clusters were evident from the figure. The most important fungi of these clusters were A. niger, A nidulans and $A$. wentii with $58.4-75.5 \%$ sequence identity with $A$. fumigatus NmrA.

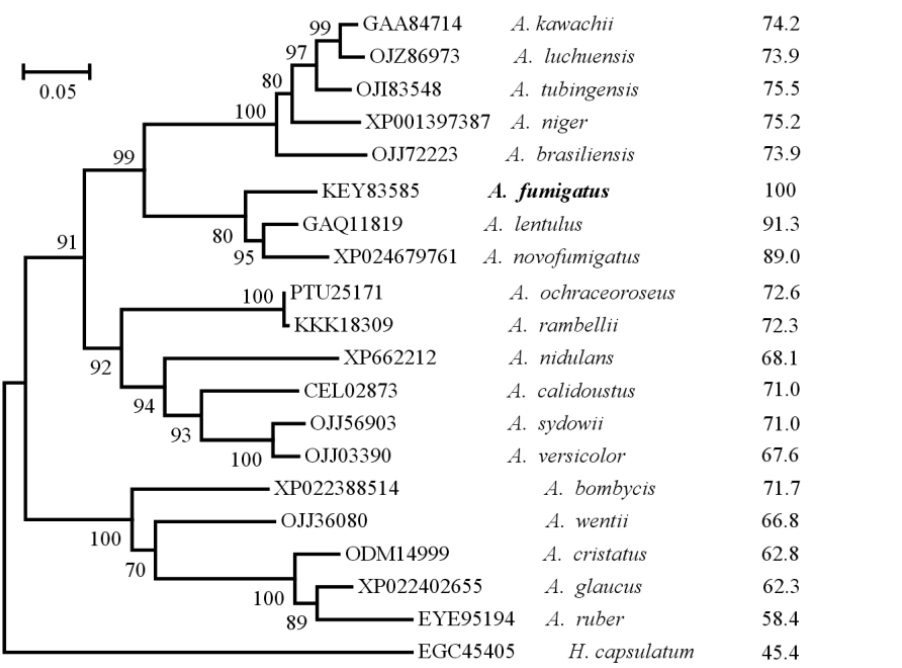

Figure 5 Consensus phylogenetic tree of NmrA of selected Aspergillus species Accession numbers are indicated for each species. NmrA of $H$. capsulatum (EGC45405) serves as outgroup. The tree is drawn to scale where the scale bar indicates percent divergence (distance) in the units of the number of amino acid substitutions per site. Evolutionary analyses were conducted in MEGA7.

\section{CONCLUSION}

Based on the quality score, the proposed structure was correct and extremely good to represent NmrA of A. fumigatus. Structural and physiochemical characterization indicated that the protein was hydrophilic in nature, stable in vitro and had very low disorder probability. Characteristic dinucleotide binding motif, SDR motif, DNA binding residues and regulatory site were also located. The protein had high sequence identity with phylogenetically close opportunistic human pathogens A. lentulus and A. novofumigatus.

\section{REFERENCES}

Abad, A., Fernández-Molina, J.V., Bikandi, J., Ramírez, A., Margareto, J., Sendino, J., ... \& Rementeria, A. (2010). What makes Aspergillus fumigatus a successful pathogen? Genes and molecules involved in invasive aspergillosis. Revista Iberoamericana de Micologia, 27, 155-182. http://dx.doi.org/10.1016/j.riam.2010.10.003

Altschul, S.F., Gish, W., Miller, W., Myers, E.W., \& Lipmas, D.J. (1990). Basic Local alignment search tool. Journal of Molecular Biology, 215, 403-410. http://dx.doi.org/10.1016/S0022-2836(05)80360-2

Andrianopoulos, A., Kourambas, S., Sharp, J.A., Davis, M.A., \& Hynes, M.J. (1998). Characterization of the Aspergillus nidulans nmrA gene involved in nitrogen metabolite repression. Journal of Bacteriology, 180, 1973-1977. https://www.ncbi.nlm.nih.gov/pubmed/107119

Balajee, S.A., Gribskov, J.L., Hanley, E., Nickle, D., \& Marr, K.A. (2005) Aspergillus lentulus sp. nov., a new sibling species of A fumigatus. Eukaryotic Cell, 4(3), 625-632. http://dx.doi.org/10.1128/EC.4.3.625-632.2005

Benkert, P., Künzli, M., \& Schwede, T. (2009). QMEAN server for protein model quality estimation. Nucleic Acids Research, 37, W510-W514 http://dx.doi.org/10.1128/EC.4.3.625-632.2005

Bongomin, F., Gago, S., Oladele, R.O., \& Denning, D.W. (2017). Global and multinational prevalence of fungal diseases-estimate precision. Journal of Fungi, 3, E57. http://dx.doi.org/10.3390/jof3040057

Cheng, J., Randall, A.Z., Sweredoski, M.J., \& Baldi, P. (2005). SCRATCH: a protein structure and structural feature prediction server. Nucleic Acids Research, 33, W72-W76. http://dx.doi.org/10.1093/nar/gki396

Cristobal, S., Zemla, A., Fischer, D., Rychlewski, L., \& Elofsson, A. (2001). A study of quality measures for protein threading models. BMC Bioinformatics, 2(1), 5. http://dx.doi.org/10.1186/1471-2105-2-5
Denning, D.W., Pashley, C., Hartl, D., Wardlaw, A., Godet, C., Del Giacco, S., .. \& Sergejeva, S. (2014). Fungal allergy in asthma-state of the art and research needs. Clinical and Translational Allergy, 4, 14. http://dx.doi.org/10.1186/20457022-4-14

Gasteiger, E., Gattiker, A., Hoogland, C., Ivanyi, I., Appel, R.D., \& Bairoch, A (2003). ExPASy: the proteomics server for in-depth protein knowledge and analysis. Nucleic Acids Research, 31, 3784-3788. https://www.ncbi.nlm.nih.gov/pubmed/12824418

Guruprasad, K., Reddy, B.V., \& Pandit, M.W. (1990). Correlation between stability of a protein and its dipeptide composition: a novel approach for predicting in vivo stability of a protein from its primary sequence. Protein Engineering, 4(2), 155-61. http://dx.doi.org/10.1093/protein/4.2.155

Hall, T.A. (1999). BioEdit: A user-friendly biological sequence alignment editor and analysis program for Windows 95/98/NT. Nucleic Acids Symposium Series 41, 95-98

Han, X., Qiu, M., Wang, B., Yin, W.B., Nie, X., Qin, Q., ... \& Wang, S. (2016) Functional analysis of the nitrogen metabolite repression regulator gene $n m r A$ in Aspergillus flavus. Frontiers in. Microbiology, 7, 1794 http://dx.doi.org/10.3389/fmicb.2016.01794

Hollingsworth, S.A., \& Karplus, P.A. (2010). A fresh look at the Ramachandran plot and the occurrence of standard structures in proteins. Biomolecular Concepts, 1(3-4), 271-283. http://dx.doi.org/10.1515/BMC.2010.022

Hsin, J., Arkhipov, A., Yin, Y., Stone, J.E., \& Schulten, K. (2008). Using VMD an introductory tutorial. Current Protocols in Bioinformatics, 5, 5.7. http://dx.doi.org/10.1002/0471250953.bi0507s24

Ikai, A. (1980). Thermostability and aliphatic index of globular proteins. Journal of Biochemistry, 88, 1895-1898 http://dx.doi.org/10.1093/oxfordjournals.jbchem.a133168

Kumar, S., Stecher, G., \& Tamura, K. (2016). MEGA7: Molecular evolutionary genetics analysis version 7.0 for bigger datasets. Molecular Biology and Evolution, 33, 1870-1874. http://dx.doi.org/10.1093/molbev/msw054

Kwon-Chung, K.J., \& Sugui, J.A. (2013). Aspergillus fumigatus-what makes the species a ubiquitous human fungal pathogen? PLoS Pathogens, 9(12), e1003743. http://dx.doi.org/10.1371/journal.ppat.1003743

Lamb, H.K., Leslie, K., Dodds, A.L., Nutley, M., Cooper, A., Johnson, C., ... \& Hawkins, A.R. (2003). The negative transcriptional regulator NmrA discriminates between oxidized and reduced dinucleotides. Journal of Biological Chemistry, 278(34), 32107-32114. http://dx.doi.org/10.1074/jbc.M304104200 Larkin, M.A., Blackshields, G., Brown, N.P., Chenna, R., Mcgettigan, P.A. Mcwilliam, H., ... \& Higgins, D.G. (2007). Clustal W and Clustal X version 2.0. Bioinformatics, 23, 2947-2948. http://dx.doi.org/10.1093/bioinformatics/btm404 Laskowski, R.A., Macarthur, M.W., Moss, D.S., \& Thornton, J.M. (1993) PROCHECK - a program to check the stereochemical quality of protein structures. Journal of Applied Crystallography, 26, 283-291. http://dx.doi.org/10.1107/S0021889892009944

Latge, J.P. (1999). Aspergillus fumigatus and aspergillosis. Clinical Microbiology Reviews, 12, 310-350. https://www.ncbi.nlm.nih.gov/pubmed/88920

Levitt, M., \& Gerstein, M. (1998). A unified statistical framework for sequence comparison and structure comparison. Proceedings of National Academy of

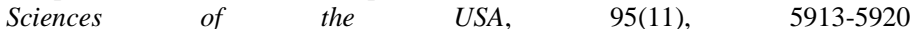
http://dx.doi.org/10.1073/pnas.95.11.5913

López-Berges, M.S., Rispail, N., Prados-Rosales, R.C., \& Dipietro, A. (2010). A nitrogen response pathway regulates virulence functions in Fusarium oxysporum via the protein kinase TOR and the bZIP protein MeaB. Plant Cell, 22, 2459-475 http://dx.doi.org/10.1105/tpc.110.075937

Macios, M., Caddick, M.X., Weglenski, P., Scazzocchio, C., \& Dzikowska, A (2012). The GATA factors AREA and AREB together with the co-repressor NMRA, negatively regulate arginine catabolism in Aspergillus nidulans in response to nitrogen and carbon source. Fungal Genetics and Biology, 49(3), 189-198. http://dx.doi.org/10.1016/j.fgb.2012.01.004

Magasanik, B., \& Kaiser, C.A. (2002). Nitrogen regulation in Saccharomyces cerevisiae. Gene, 290(1-2), 1-18. http://dx.doi.org/10.1016/S03781119(02)00558-9

Marchler-Bauer, A., Derbyshire, M.K., Gonzales, N.R., Lu, S., Chitsaz, F., Geer L.Y., .. \& Bryant, S.H. (2017). CDD/SPARCLE: functional classification of proteins via subfamily domain architectures. Nucleic Acids Research, 43, 222 226. http://dx.doi.org/10.1093/nar/gke1129

Mulichak, AM., Theisen, M.J., Essigmann, B., Benning, C., \& Garavito, R.M (1999). Crystal structure of SQD1, an enzyme involved in the biosynthesis of the plant sulfolipid headgroup donor UDP-sulfoquinovose. Proceedings of National Academy of Sciences of the USA, 96(23), 13097-13102. http://dx.doi.org/10.1073/pnas.96.23.13097

Nierman, W.C., Pain, A., Anderson, M.J., Wortman, J.R., Kim, H.S., Arroyo, J., ... \& Denning, D.W. (2005). Genomic sequence of the pathogenic and allergenic filamentous fungus Aspergillus fumigatus. Nature, 438, 1151-1156. http://dx.doi.org/10.1038/nature04332

Nichols, C.E., Cocklin, S., Dodds, A., Ren, J., Lamb, H., Hawkins, A.R., \& Stammers, D.K. (2001). Expression, purification and crystallization of Aspergillus nidulans NmrA, a negative regulatory protein involved in nitrogenmetabolite repression. Acta Crystallographica Section D Biological 
Peláez, T., Alvarez-Pérez, S., Mellado, E., Serrano, D., Valerio, M., Blanco, J.L., ... \& Bouza, E. (2013). Invasive aspergillosis caused by cryptic Aspergillus species: a report of two consecutive episodes in a patient with leukaemia. Journal

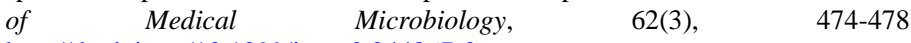
http://dx.doi.org/10.1099/jmm.0.044867-0

Prilusky, J., Felder, C.E., Zeev-Ben-Mordehai, T., Rydberg, E.H., Man, O., Beckmann, J.S., ... \& Sussman, J.L. (2005). FoldIndex: a simple tool to predict whether a given protein sequence is intrinsically unfolded. Bioinformatics, 21(16), 3435-3438. http://doi.org/10.1093/bioinformatics/bti537

Saito, N., \& Nei, M. (1987). The neighbor-joining method: a new method for reconstructing phylogenetic trees. Molecular Biology and Evolution, 4, 406-425. http://dx.doi.org/10.1093/oxfordjournals.molbev.a040454

Siew, N., Elofsson, A., Rychlewski, L., \& Fischer, D. (2000). MaxSub: an automated measure for the assessment of protein structure prediction quality. Bioinformatics, 16(9), 776-785.

http://dx.doi.org/10.1093/bioinformatics/16.9.776

Stammers, D.K., Ren, J., Leslie, K., Nichols, C.E., Lamb, H.K., Cocklin, S., ... \& Hawkins, A.R. (2001). The structure of the negative transcriptional regulator NmrA reveals a structural superfamily which includes the short-chain dehydrogenase/reductases. EMBO Journal, 20(23), 6619-6626. http://dx.doi.org/10.1093/emboj/20.23.6619

Szklarczyk, D., Morris, J.H., Cook, H., Kuhn, M., Wyder, S., Simonovic, M., .. \& Von Mering, C. (2017). The STRING database in 2017: quality-controlled protein-protein association networks, made broadly accessible. Nucleic Acids Research, 45, D362-D368. http://dx.doi.org/10.1093/nar/gkw937

Tjong, H., \& Zhou, H.X. (2007). DISPLAR: an accurate method for predicting DNA-binding sites on protein surfaces. Nucleic Acids Research, 35, 1465-1477. http://dx.doi.org/10.1093/nar/gkm008

Tudzynski, B. 2014. Nitrogen regulation of fungal secondary metabolism in fungi. Frontiers in Microbiology, 5, 656. http://dx.doi.org/10.3389/fmicb.2014. $\underline{00656}$

Wagner, D., Schmeinck, A., Mos, M., Morozov, I.Y., Caddick, M.X., \& Tudzynski, B. (2010). The bZIP transcription factor MeaB mediates nitrogen metabolite repression at specific loci. Eukaryotic Cell, 9, 1588-1601. http://dx.doi.org/10.1128/EC.00146-10

Wallner, B., \& Elofsson, A. (2003). Can correct protein models be identified? Protein Science, 12(5), 1073-1086. http://dx.doi.org/10.1110/ps.0236803

Willard, L., Ranjan, A., Zhang, H., Monzavi, H., Boyko, R.F., Sykes, B.D., \& Wishart, D.S. (2003). VADAR: a web server for quantitative evaluation of protein structure quality. Nucleic Acids Research, 31(13), 3316-3319. http://dx.doi.org/10.1093/nar/gkg565

Wilson, R.A., \& Arst, H.N. Jr. (1998). Mutational analysis of AREA, a transcriptional activator mediating nitrogen metabolite repression in Aspergillus nidulans and a member of the 'streetwise' GATA family of transcription factors. Microbiology and Molecular Biology Review, 62, 586-596. https://www.ncbi.nlm.nih.gov/pubmed/9729601

Wong, K.H., Hynes, M.J., Todd, R.B., \& Davis, M.A. (2007). Transcriptiona control of nmrA by the bZIP transcriptional factor MeaB reveals a new level of nitrogen regulation in Aspergillus nidulans. Molecular Microbiology, 66, 534551. http://dx.doi.org/10.1111/j.1365-2958.2007.05940.x

Zuckerkandl, I., \& Pauling L. (1965). Evolutionary divergence and convergence in proteins. In V. Bryson \& H.J. Vogel (Ed.), Evolving genes and proteins. Academic Press, New York. pp. 97-166. ISBN 9781483266305. http://dx.doi.org/10.1016/B978-1-4832-2734-4.50017-6 